Service social

\title{
Une démarche de recherche-action pour l'analyse de la situation et des besoins des femmes dans Lotbinière
}

\section{Françoise Roinsol}

Volume 34, numéro 2-3, 1985

L'organisation communautaire

URI : https://id.erudit.org/iderudit/706273ar

DOI : https://doi.org/10.7202/706273ar

Aller au sommaire du numéro

Éditeur(s)

École de service social de l'Université Laval

ISSN

1708-1734 (numérique)

Découvrir la revue

Citer cet article

Roinsol, F. (1985). Une démarche de recherche-action pour l'analyse de la situation et des besoins des femmes dans Lotbinière. Service social, 34(2-3), 294-299. https://doi.org/10.7202/706273ar d'utilisation que vous pouvez consulter en ligne. 
RoInsol, Françoise, chargée de cours à I'Université Memorial, Saint-Jean de Terre-Neuve.

\section{Une démarche de recherche-action pour l'analyse de la situation et des besoins des femmes dans Lotbinière}

\section{Françoise Roinsol *}

Répondant aux demandes formulées plusieurs fois par des femmes de la région, le Centre local de services communautaires (C.L.S.C.) Arthur-Caux donnait mandat, à l'automne 1983, à une organisatrice communautaire d'évaluer les besoins des femmes de la région et de proposer des pistes de solutions aux problèmes identifiés. En effet, bien que les usagers du C.L.S.C. soient très majoritairement de sexe féminin, l'organisme n'avait jamais entrepris de réflexion ou d'action concertée face à cette clientèle. On voulait vérifier si les services offerts répondaient bien aux besoins ressentis prioritairement par celles-ci, et s'il y avait d'autres dynamiques à mettre en marche pour que les groupes du milieu et les femmes elles-mêmes prennent en charge certains besoins.

Face à cette demande, nous avons proposé au C.L.S.C. d'effectuer une démarche de recherche-action qui permettrait aux femmes du milieu d'exprimer elles-mêmes leur situation, leurs difficultés, leurs besoins. Nous nous sommes inspirée de la méthode de l'enquête conscientisante ${ }^{1}$ pour mener, pendant deux ans, une démarche avec un groupe de femmes de la région.

\section{Constitution de l'équipe}

À la suite d'un appel lancé auprès des différentes associations du milieu (Cercles de fermières, clubs de l'âge d'or, groupes populaires, etc.), et par l'intermédiaire de l'hebdomadaire régional, une douzaine de personnes ont accepté de devenir "enquêteuses". Elles y voyaient une façon concrète d'aider les femmes de la région tout en vivant une 
expérience enrichissante pour elles-mêmes. Pendant plus de deux ans, ce groupe se réunira une demi-journée par semaine.

\section{Élaboration du questionnaire}

Les thèmes et questions abordés dans cet instrument ont été retenus à partir de l'inventaire des difficultés, besoins et problèmes des femmes tels que perçus par les personnes travaillant au C.L.S.C. et par les membres du groupe. Ces deux analyses ont permis de retenir douze thèmes : travail, économie, famille, sexualité et contraception, santé physique et mentale, formation et socialisation, vie sociale, isolement, peurs, stéréotypes et préjugés, pornographie, violence. La préparation du questionnaire définitif, comprenant quatre-vingt-seize questions, a demandé près de quatre mois de travail.

\section{Application du questionnaire}

Pour être sûres de contacter des femmes de tous âges et de toutes catégories sociales, l'échantillon des personnes interrogées a été constitué en prenant une personne sur vingt à l'intérieur de la liste électorale. Chacune d'elle a reçu une lettre la prévenant qu'elle avait été choisie et qu'une enquêteuse la contacterait pour prendre un rendez-vous. Les questionnaires ont donc été distribués de main à main par les enquêteuses qui en profitaient pour expliquer la démarche et les objectifs de l'enquête aux femmes. Celles-ci recevaient en même temps une enveloppe pré-adressée et affranchie pour le retour du questionnaire. L'anonymat des réponses était ainsi garanti.

De manière générale, les enquêteuses ont reçu un excellent accueil; la plupart des femmes choisies se sont montrées intéressées, heureuses que l'on se préoccupe enfin de leur situation particulière en milieu rural et, pour un certain nombre, flattées d'avoir été choisies pour y participer.

\section{Quelques données de l'enquête}

À la lumière des résultats compilés à partir des deux cent vingt questionnaires reçus, nous pouvons dire que rien de très inattendu n'est ressorti de cette enquête. La situation des femmes dans le comté de Lotbinière paraît bien similaire à celle existant dans les villes et les autres régions du Québec et du Canada. Près de la moitié d'entre elles ont un emploi rémunéré; la plupart ne sont pas économiquement 
autonomes; le quart d'entre elles avouent avoir déjà été victimes de violence (principalement dans la famille) ; elles sont pratiquement les seules responsables des tâches ménagères et des soins aux enfants, etc.

Les quelques données plus spécifiques au milieu rural sont: l'enracinement (le plus grand nombre habite la région depuis plus de vingt ans), la force des préjugés qui les oblige à adopter des comportements traditionnels (se marier, aller à l'église), la surexploitation des couturières à domicile (qui gagnent entre $0,30 \$$ et $1,50 \$$ l'heure), et la forte présence de la pornographie (entre autres parce qu'il n'y a pas d'autres lieux de sorties que les bars).

\section{Présentation des résultats}

Les réponses au questionnaire ont été regroupées en quatre cahiers thématiques dans lesquels ont été retranscrites les données quantifiables ainsi que les réponses aux questions ouvertes. Certaines données significatives ont été mises en valeur par un encadré ou par une illustration. Ces documents ont été très largement diffusés aux personnes intéressées.

De plus, l'équipe des enquêteuses a produit un diaporama d'une durée de quinze minutes destiné à présenter à la population les résultats de l'enquête et se terminant par un appel pour que des actions concrètes suivent le constat présenté.

Enfin, des affiches illustrant les thèmes sur lesquels l'équipe voulait particulièrement interpeller la population ont été réalisées et plastifiées afin d'être exposées dans les salles où ont eu lieu les débats publics.

\section{Conférence de presse}

Le 15 novembre 1984, l'équipe des enquêteuses a organisé une conférence de presse à laquelle étaient invités, outre la presse locale et régionale, les représentants des groupes sociaux de Lotbinière et les groupes de femmes de la région. Une trentaine de personnes y ont participé et, ayant été très bien couverte par les médias, elle a permis au groupe de faire connaître les grandes lignes des résultats de l'enquête, et surtout d'annoncer à la population la tenue prochaine, dans les différentes paroisses du comté, de débats publics autour de ces résultats. 


\section{Rencontres publiques}

Tout au long de l'hiver 1985, l'équipe des enquêteuses a rencontré la population au cours de vingt-cinq réunions qui ont permis d'échanger avec près de sept cents personnes sur les résultats de l'enquête et les suites à y donner.

Il convient de noter l'aide précieuse qu'a été la collaboration des groupes sociaux, principalement les Cercles des fermières, qui nous ont reçues à l'intérieur de leurs réunions régulières, nous permettant ainsi de rejoindre un grand nombre de femmes.

Les rencontres, d'une durée moyenne de deux heures, comprenaient, après le visionnement du diaporama, des échanges en ateliers thématiques (lorsqu'il y avait plus de douze personnes), suivis d'une plénière qui permettait de mettre en commun les principaux problèmes identifiés et d'échanger sur les actions à entreprendre.

Toutes ces rencontres ont été animées par des enquêteuses qui avaient reçu une formation adéquate, car peu avaient déjà de l'expérience en animation.

\section{Pour assurer des suites : la création d'un Centre-Femmes}

Afin d'améliorer leur situation dans le comté, de nombreuses actions, suggérées lors des rencontres publiques, nécessitaient l'existence d'un lieu commun aux femmes de la région et pouvant servir de point de départ à l'organisation d'activités. C'est ainsi qu'est née, petit à petit, l'idée d'un Centre-Femmes pour la région de Lotbinière. L'équipe des enquêteuses étant très motivée à ce que tout le travail réalisé depuis plus de deux ans ne reste pas lettre morte, a donc repris cette idée très rapidement. Un projet «Canada au travail » a été formulé puis obtenu, ce qui a permis à quatre des douze membres de l'équipe de pouvoir travailler à temps plein, pendant quatre mois, sur ce projet.

Créé pour répondre aux demandes des femmes du comté, le Centre-Femmes est donc essentiellement un lieu de rencontre, d'échange, d'information et d'écoute. II ne prétend pas apporter la solution aux problèmes qu'elles vivent (dépendance économique, violence, exploitation, etc.), mais compte leur permettre de sortir de leur isolement, de mieux connaitre leurs droits et les ressources existantes, et surtout de vivre une solidarité indispensable à l'élimination de l'oppression des femmes dans la société. 


\section{Le rôle de l'organisatrice communautaire}

Tout au long de la démarche décrite plus haut, le C.L.S.C. a apporté son soutien au groupe de femmes par l'intermédiaire d'une organisatrice communautaire.

Pendant l'enquête, celle-ci avait un rôle prépondérant de direction méthodologique et d'animation du groupe. Cela a demandé près de quatre jours par semaine durant un an et demi. Les enquêteuses fournissaient alors une demi-journée de travail, entièrement bénévole, par semaine.

À l'automne 1984, soit après un an de travail, le groupe, ayant les résultats de l'enquête, en est arrivé à la phase de présentation de ces résultats par la rédaction des "cahiers thématiques", la réalisation du diaporama et la préparation des réunions publiques. Ce travail a été encadré par l'organisatrice communautaire mais, à partir de ce moment, les participantes étaient rémunérées pour leur travail grâce à une subvention du Bureau de promotion de la femme du Secrétariat d'État, et certaines $y$ ont investi plusieurs jours par semaine.

La troisième phase, l'organisation et l'animation des débats publics, a été prise en charge et réalisée presque entièrement par les femmes elles-mêmes. L'organisatrice communautaire n'a animé aucune de ces rencontres et se limitait à participer à la réunion hebdomadaire du groupe.

Enfin, la dernière étape, donc la mise sur pied du Centre-Femmes, a été essentiellement l'affaire du groupe, composé, depuis février 1985 , de quatre permanentes payées à temps plein, et de quatre autres bénévoles. L'organisatrice communautaire participait alors comme personne-ressource à la réunion hebdomadaire animée par une des permanentes et supervisait administrativement le projet "Canada au travail».

\section{La démarche des enquêteuses}

La grande majorité des douze personnes qui se sont engagées dès le début de cette démarche étaient venues y chercher un lieu où elles pourraient échanger, à l'intérieur d'un projet tourné vers l'action et pour d'autres femmes.

Sauf les deux plus jeunes, qui s'affichaient résolument féministes, les autres étaient assez représentatives de l'idéologie traditionnelle. Leur engagement répondait à un besoin de sortir de l'isolement et d'avoir une activité valorisante qui leur permettrait d'apprendre et de recevoir autant que de donner. Après un an de travail bénévole, 
plusieurs ont signifié leur intention de se chercher un emploi rémunéré. C'est ce qui a amené le groupe à faire des démarches pour trouver du financement afin de les garder.

Quelques femmes ont quitté le groupe en cours de route, soit pour des raisons personnelles, soit pour prendre un travail rémunéré ; mais d'autres les ont remplacées, ce qui fait que l'équipe a été constamment formée de douze membres.

\section{Conclusion}

L'ensemble de cette démarche prouve que, lorsqu'on lui fournit du support, la population est capable d'exprimer ses besoins et de se donner des moyens d'y répondre. À partir d'un projet d'analyse des besoins formulés dans les bureaux d'un C.L.S.C., on a abouti, en deux ans, à un Centre-Femmes entièrement autonome et pris en charge par les femmes du milieu. En mettant sur pied ce lieu de solidarité, c'est un premier outil qu'elles se sont donné afin d'apporter des éléments de solutions aux difficultés qu'elles vivent.

\section{Notes}

* Organisatrice communautaire au C.L.S.C. Arthur-Caux, de Laurier-Station, de 1983 à 1985.

1 Humbert, Colette et Jean Merlo, L'enquête conscientisante - Problèmes et méthodes, Paris, L'Harmattan, 1978. ("INODEP: Document de travail», 5.) 